\title{
Operation and Improvement on Highway Toll Plaza
}

\author{
Feiliang $\mathrm{Hu}$ \\ Department of Electronic and Communication Engineering, North China Electric Power University, \\ Baoding 071003, China; \\ www.734844143@qq.com
}

\begin{abstract}
In working out a model that would make optimization of the feature of toll plaza, such as shape, size and merging-pattern, our team decided to apply the Queuing Theory and simulation software Vissim to figure out parameters of microcosmic traffic model .Studying on a large sum of relevant data, we firstly select average waiting time in $\mathrm{M} / \mathrm{M} / \mathrm{B}$ theory and the area of toll plaza to establish objected function to evaluate our queue model. So we record the veh of the Great Egg Toll Plaza. Considering traffic loads, we give the solution to open CAR and HGV mixed lanes to improve vehicle throughput of toll plaza when light loads. What's more, we give different solutions to fix our model to fit different situation such as self-driving and accident prevention. Last but not least, we establish models in order to persuade more states to adopt models to realize traffic jam remission and protect environment.
\end{abstract}

Keywords: Queuing Theory, Vissim simulation optimization.

\section{Introduction}

When exiting the tollbooths in a barrier toll, vehicles must "fan in" from the larger number of tollbooth egress lanes to the smaller number of regular travel lanes. A toll plaza is the area of the highway needed to facilitate the barrier toll, consisting of the fan-out area before the barrier toll, the toll barrier itself, and the fan-in area after the toll barrier.

Three types of tollbooths such as conventional tollbooths, exact-change tollbooths, and electronic toll collection booths.

\section{Solution}

We discuss the queuing model before tollbooths and merging pattern after toll. What's more, we need establish model and select parameters to evaluate the performance of our models.

\section{Establish M/M/B Model and Optimize the Proportion of Different Tollbooth Types}

Considering the toll plaza is not only one type of toll station, each type of tollbooth has different parameters, so we set up a M/M/B model for multi-toll plaza-model.

We select parameters such as average serve time, average

wating time and expense, we set the objective function:

$$
\operatorname{Minf}(\mathrm{x})=\left(\sum_{i=1}^{3} \varphi_{i} W_{i}\right) S
$$

Explanation : $\varphi_{i}$ is the weight of different toll types choosen, (i=1,2,3), respectively represent Manual, Coin-Bin and E-ZPass

Referring to the Usage of E-Zpass.

According to the Port Authority of New York and New Jersey, 83.2\% of vehicles crossing its five bridges and tunnels used E-ZPass for toll pay-ment during the first half of 2016 [1]. 
We set reasonable value of $\varphi_{i}, \mathrm{~B}, \mathrm{~L}$ as a real example:

$$
\varphi_{i}=\left[\begin{array}{lll}
0.1 & 0.1 & 0.8
\end{array}\right], \mathrm{B}=8, \mathrm{~L}=3
$$

Based on the common statistics, we make a assumption that the Car accounted for $70 \%$ and $\mathrm{HGV}$ account for $30 \%$

According to the standard for highway [2] (table 1).

Table 1. $\lambda_{s}=v p h / 3600($ cars $/ s)$

\begin{tabular}{|c|c|c|}
\hline Method & Car Throughput vph & HGV Throughput vph \\
\hline E-ZPass & $450-900$ & $300-500$ \\
\hline Coin-Bin & $300-500$ & $200-350$ \\
\hline Manual & $250-550$ & $200-300$ \\
\hline
\end{tabular}

We find a formula for calculating C--the throughput of the toll plaza(cars/s):

$$
\begin{aligned}
& C=N \cdot\left(P_{k} C_{k}+P_{h} C_{h}\right) \\
& C=N \cdot\left(P_{k} C_{k}+P_{h} C_{h}\right)
\end{aligned}
$$

Where, $\mathrm{N}$ is the number of toll lanes, $P_{k}$ is proportion of car, $P_{h}$ is proportion of $\mathrm{HGV}, C_{h}$ is conversion coefficient for $\mathrm{HGV}, C_{k}$ is conversion coefficient for car.

\section{Common Merging Pattern to Multi-merging-pattern}

As shown at Figure 1 on the left side of the square for the original shape, the right side of the initi al improvement for us to shape, we do this is to improve from the previous merger into the two mer ger.

We define the difficulty of the merger $\mathrm{O}$, then we can get:

$$
\mathrm{O}=\mathrm{L} / \mathrm{B}
$$

previous difficulty is

$$
O(1)=\frac{L}{B}
$$

After improving,

$$
O(n)=\prod_{i=1}^{n} \frac{L_{i}}{B_{i}} \alpha i
$$

Obviously $\mathrm{O} 2<\mathrm{O} 1$, therefore, when $\mathrm{n}$ approaches infinity, we get the minimum of $\mathrm{O}(\mathrm{n})$ and toll plaza boundary approaches a curve.At the same time, we find that expense increases following area.So we consider using quadratic function to fit the curve.Setting $\mathrm{Sn}$ as the area of our new design,we need optimize function $g(x)=S n \cdot O(n)$, the matrix of toll types is [5] (figure 2 and figure 3). 


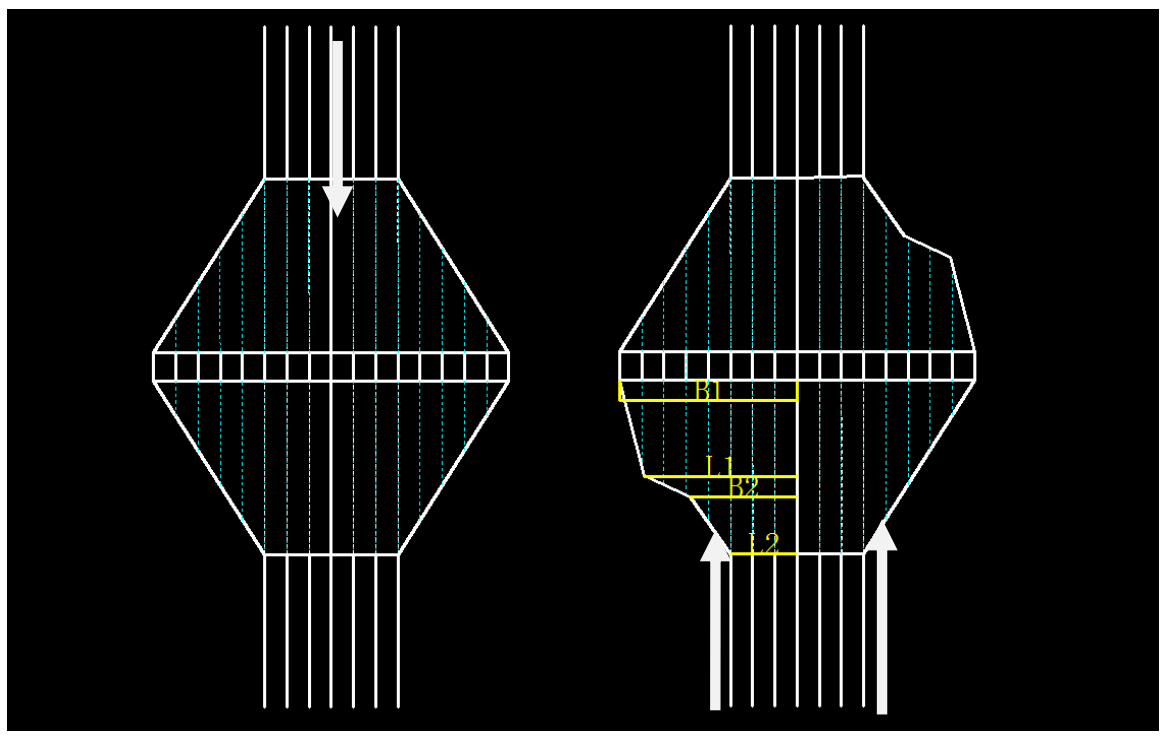

Fig.1 matrix of toll types

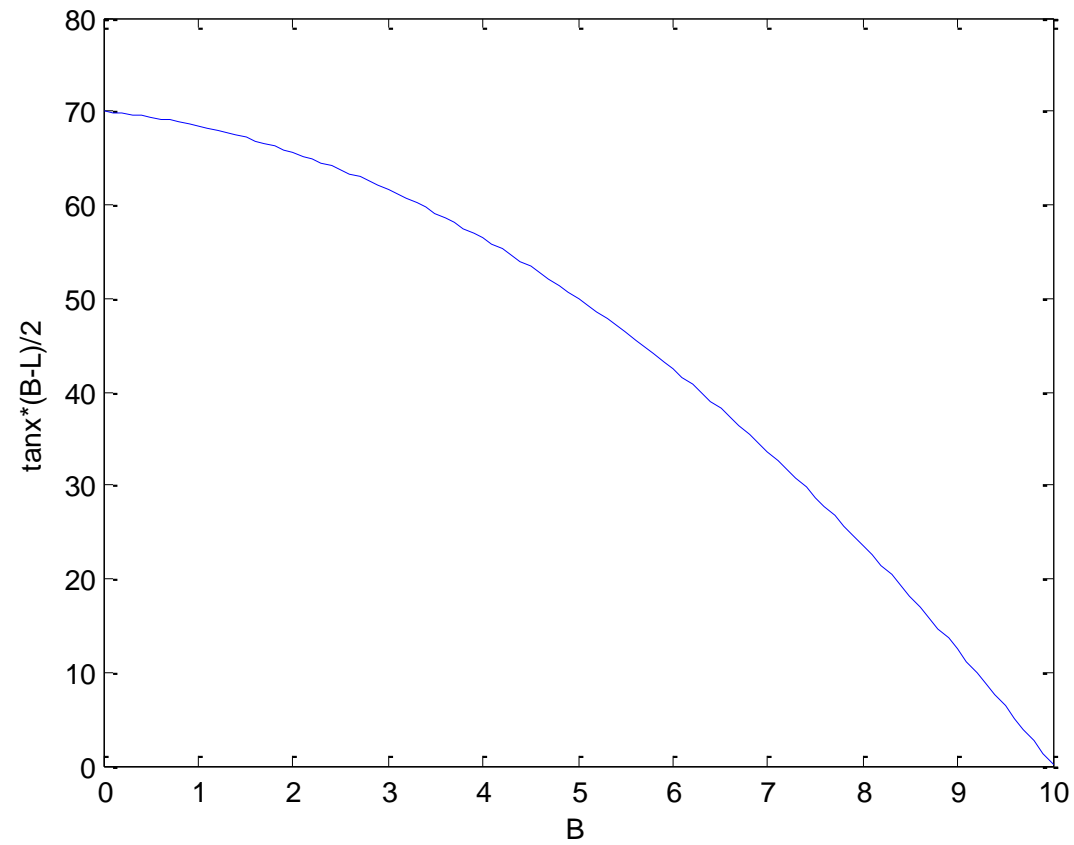

Fig. 2 matrix of toll types

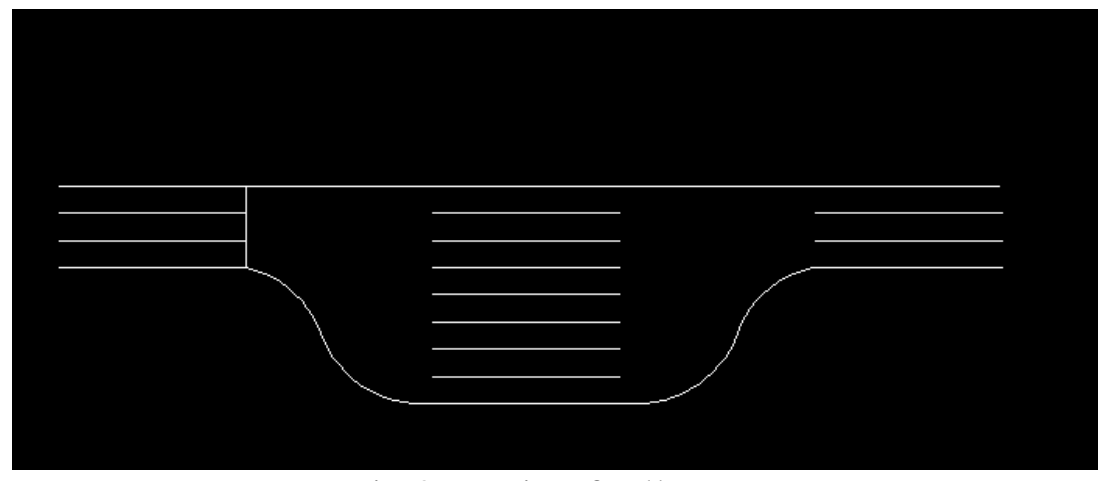

Fig.3 matrix of toll types 


\section{Simulating (table 2)}

Table 2 simulation

\begin{tabular}{|c|c|c|}
\hline Constant & Symbol & Value \\
\hline acceleration & $\mathrm{a}$ & \\
\hline deceleration & $\mathrm{a}$ & $3.5 \mathrm{~m}$ \\
\hline lane width & wd1 & $28 \mathrm{~m} / \mathrm{s}$ \\
\hline speed limit & Vmax & $2.8 \mathrm{~m}$ \\
\hline toll lane width & wd2 \\
\hline
\end{tabular}

Light traffic loads is shown in figure 4.

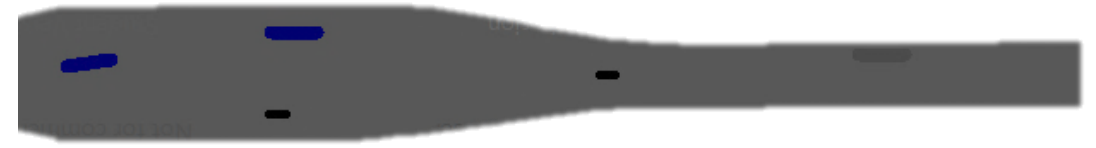

Fig.4 light traffic loads

Due to the appearance of self-driving, we can consider the relevance between self-driving and ETC toll type. We assume that the proportion of unmanned vehicles is $\Lambda$, on the other hand, that of ordinary vehicles is $1-\Lambda$, we assume that autonomous vehicles use ETC and ordinary vehicles use three types of tollbooths.

Here we will set $\Lambda$ as three cases of $0.2,0.4,0.8$. We use the $\mathrm{M} / \mathrm{M} / \mathrm{B}$ model, make correction respectively for $\phi \mathrm{i}$ :

$$
\text { [0.08 } 0.08 \text { 0.24], [0.06 } 0.060 .88 \text {, [0.02 } 0.020 .96 \text { ] }
$$

The numbers of ETC were 2, 3, 5, by the data we can get a positive correlation with ETC, and the remaining two were negatively correlated.

\section{Conclusion}

In working out a model that would make optimization of the feature of toll plaza, such as shape, size and merging-pattern, our team decided to apply the Queuing Theory and simulation software Vissim to figure out parameters of microcosmic traffic model .Studying on a large sum of relevant data, we firstly select average waiting time in M/M/B theory and the area of toll plaza to establish objected function to evaluate our queue model. So we record the veh of the Great Egg Toll Plaza. Considering traffic loads, we give the solution to open CAR and HGV mixed lanes to improve vehicle throughput of toll plaza when light loads. What's more, we give different solutions to fix our model to fit different situation such as self-driving and accident prevention. Last but not least, we establish models in order to persuade more states to adopt models to realize traffic jam remission and protect environment.

\section{References}

[1]. Information on https://en.wikipedia.org/wiki/E-ZPass.

[2]. Information on www.standardsforhighways.co.uk_ha_standards_dmrb_vol6_section3_ta9808. 